\title{
Multiple ultrasound cavitation-enabled treatments for myocardial reduction
}

\author{
Douglas L. Miller ${ }^{1,3^{*}}$, Xiaofang Lu', Chunyan Dou', Yiying I. Zhu', Mario L. Fabiilli', Gabe E. Owens ${ }^{2}$ \\ and Oliver D. Kripfgans ${ }^{1}$
}

\begin{abstract}
Background: Ultrasound myocardial cavitation enabled treatment (MCET) is an image-guided method for tissue reduction. In this study, a strategy of fractionated (multiple) treatments was tested for efficacy.

Methods: Dahl SS rats were anesthetized and prepared for treatment with a focused ultrasound transducer in a warm water bath. Aiming at the anterior left ventricular wall was facilitated by imaging with a $10 \mathrm{MHz}$ phased array (10S, GE Vivid 7, GE Vingmed Ultrasound, Horten, Norway). MCET was accomplished at $1.5 \mathrm{MHz}$ by pulse bursts of $4 \mathrm{MPa}$ peak rarefactional pressure amplitude, which were intermittently triggered 1:8 from the ECG during infusion of a microbubble suspension for cavitation nucleation. Test groups were sham, a $200 \mathrm{~s}$ treatment, three $200 \mathrm{~s}$ treatments a week apart, and a $600 \mathrm{~s}$ treatment. Treatment outcome was observed by plasma troponin after $4 \mathrm{~h}$, echocardiographic monitoring and histology at $6 \mathrm{wk}$.
\end{abstract}

Results: The impacts of the fractionated treatments summed to approximately the same as the long treatment; e. $\mathrm{g}$. the troponin result was $10.5 \pm 3.2$ for 200 s, $22.7 \pm 5.4(p<0.001)$ for the summed fractionated treatments and 29 . $9 \pm 6.4$ for $600 \mathrm{~s}$ ( $p=0.06$ relative to the summed fractionated). While wall thickness was not reduced for the fractionated treatment, tissue strain was reduced by $35 \%$ in the target area relative sham $(p<0.001)$.

Conclusion: The ability to fractionate treatment may be advantageous for optimizing patient outcome relative to all-or nothing therapy by surgical myectomy or alcohol ablation.

Keywords: Myocardial contrast echocardiography, Arrhythmia, Cardiac myocyte necrosis, Hypertrophic cardiomyopathy

\section{Background}

Hypertrophic cardiomyopathy (HCM) is a relatively common genetic cardiovascular disease, occurring in more than 1 in 500 people $[1,2]$. HCM can occur in several regions of the myocardium, and is particularly troubling when restrictions occur in the left ventricular outflow tract (LVOT). Patients may experience shortness of breath, angina, palpitations, and even sudden death in young athletes [3]. Hypertrophy leads to obstruction of the LVOT in up to $75 \%$ of patients, either at rest or with exercise. One-third of patients with obstruction remain symptomatic after pharmacological therapy and are candidates for myocardial reduction [4]. Apical hypertrophy

\footnotetext{
*Correspondence: douglm@umich.edu

'Department of Radiology, University of Michigan School of Medicine, Ann Arbor, MI 48109, USA

${ }^{3}$ University of Michigan School of Medicine, 3240A Medical Sciences Building I, 1301 Catherine Street, Ann Arbor, Ml 48109-5667, USA

Full list of author information is available at the end of the article
}

is also troubling and currently has no available corrective therapy [5].

Surgical septal myectomy is presently the primary method for reduction of asymmetric septal hypertrophy with perturbation of the mitral valve leaflets $[6,7]$. This invasive procedure simply deletes a volume of the myocardium to relieve the obstruction. For HCM patients with surgical myectomy, the interventricular wall thickness was reduced by $18 \%$ from 19.4 to $15.9 \mathrm{~mm}$ [8]. Transcatheter septal ablation with alcohol has been developed as an alternative to surgery [7], and has achieved some success in terms of safety and efficacy [9]. The immediate therapeutic benefit appears to depend on akinesis of the effected region, rather than an immediate reduction in size. However, septal thinning develops gradually after alcohol treatment and reductions from 23.7 to $18.0 \mathrm{~mm}$ (24\%) have been reported after 6 months [10]. The alcohol procedure results in a 
volume of complete necrosis, which progresses to myocardial fibrosis and scar [11]. This procedure has a high incidence of heart block (about 20\%) requiring permanent pacemaker [4], and healing of the alcohol infarction to massive scar carries a risk of serious arrhythmia [12]. These procedures are comparable with low rates in overall mortality and sudden cardiac death after treatment [9].

An alternative to myectomy or alcohol ablation would be a useful additional option for some patients if it were less invasive or more precise in creation of myocardial reduction or akinesis, and could be utilized for apical or other presentations of hypertrophy not suitable for the established treatments. We have developed a novel ultrasonic method of myocardial treatment, which employs intermittent pulsed ultrasound exposure of the myocardium during infusion of a microbubble contrast agent. Infused microbubbles are destroyed by relatively high pressure amplitude pulses, and the intermittent delay allows capillaries to refill with blood containing fresh microbubbles. The microbubble destruction nucleates ultrasonic cavitation within the focal zone, leading to randomly scattered microlesions each with capillary injury and one or a few lethally injured cardiomyocytes. This nonthermal myocardial cavitation-enabled treatment (MCET) method was based on the diagnostic myocardial contrast echocardiography technique using contrast destructive pulses with observation of contrast refill for perfusion assessment. Scattered cardiomyocyte injury was detected for myocardial contrast echocardiography [13], but MCET employs higher than diagnostic pressure amplitudes for therapeutic myocardial reduction [14]. This treatment produces microlesions which accumulate with each intermittent pulse allowing adjustment of microlesion density from modest fractions of tissue volume to more dense distributions within a targeted focal zone [15]. The effected region can be accurately created by the image-guided placement of the ultrasonic focus. Echocardiography is the gold standard method for HCM diagnosis and treatment follow up. The combination of MCET with diagnostic imaging presents a compelling new concept of non-invasive and highly controlled HCM therapy.

In studies with rats, MCET has utilized a small $1.5 \mathrm{MHz}$ focused transducer aimed with guidance from a $10 \mathrm{MHz}$ cardiac probe on a GE Vivid 7 Dimension ultrasound machine [14]. Highly effective treatment involves 10 cycle pulses of $4 \mathrm{MPa}$ peak rarefactional pressure amplitude delivered intermittently each 8 heartbeats during continuous IV infusion of microbubble contrast agent suspension for 10 min of lesion accumulation. Most pulse-triggers produced premature complexes (PCs) in the ECG, when triggered at the end of systole, which are indicative of treatment progress [16]. If pulse triggers produce few PCs, then the resulting treatment effect will be inadequate. Troponin I measurements in blood samples provide an overall measure of treatment impact. Wall thickness and wall strain and displacement are measured by echocardiography, and fibrosis is assessed in histology.

Longer-term maturation of the myocardial volumes with MCET microlesions was studied to assess the potential therapeutic effect in normal Dahl-SS rats [17], and in the SS-16 ${ }^{\mathrm{BN}}$ rat model of HCM [18]. The consomic Dahl SS rat with chromosome 16 from brown Norway rats develops progressive left ventricular (LV) hypertrophy without hypertension [19]. For normal rats, significant inflammatory response to the injury increased the heart wall thickness the day after treatment, as measured by echocardiography [17]. After 4 weeks, no significant tissue reduction was noted in the treated region with scattered areas of tissue fibrosis. For the HCM model rats, the inflammatory response was greatly reduced by adjuvant steroid given at treatment and adjuvant treatment with an angiotensin II receptor blocker was used to minimize scarring [18]. MCET rapidly produced a significant reduction in myocardial strain rate and endocardial displacement. At $28 \mathrm{~d}$ post therapy, the targeted wall thickness was significantly reduced by $16.2 \%(p<0.01)$ relative to shams, and strain rate and displacement were, reduced by $34 \%$ and $29 \%$, respectively, which are sufficient for therapeutic treatment.

An important advantage of MCET, for example, relative to alcohol ablation, is the ability to monitor outcome by echocardiography, and re-treat desired segments of the myocardium by image guided placement of the focal zone. This is limited by the depth of the focal zone, so that in rats, the $1.5 \mathrm{MHz}$ focal zone passes through the heart giving lesions on the anterior, posterior and septal regions. A system designed for MCET in humans would have the ability to target desired regions more specifically, and to rapidly treat by intermittent scans. Another advantageous feature is the ability to gradually accumulate microlesions to form a desired "macrolesion" treatment impact. A conservative treatment could be augmented at a later date and adjusted in shape and size as desired. This present study was designed to test the idea of multiple treatment accumulation using the normal rat model. Three treatments of $200 \mathrm{~s}$, spaced a week apart, were compared to 3 sham $200 \mathrm{~s}$ treatments, one $200 \mathrm{~s}$ treatment and one $600 \mathrm{~s}$ treatment (i. e., the 10 min highly efficacious protocol).

\section{Methods}

\section{Animal preparation}

All in vivo animal procedures were conducted with the approval and guidance of the University Committee on Use and Care of Animals of the University of Michigan. Normal Dahl SS rats (Charles River, Wilmington, MA, 
USA) were used for this study for comparison to earlier work [14, 17]. For ultrasound treatment, the rats were anesthetized by intraperitoneal injection of ketamine $\left(90 \mathrm{mg} \mathrm{kg}^{-1}\right)$ and xylazine $\left(9 \mathrm{ml} \mathrm{kg}^{-1}\right)$ and the left thorax was shaved and depilated for ultrasound transmission to the heart. A 24 gauge cannula (BD Angiocath, Becton Dickinson Infusion Therapy Systems, Sandy, UT, USA) was inserted into the tail vein for intravenous injections of contrast agent. The rats were mounted on a positioning board and needle electrodes were placed in the forelegs and left hind leg for detection of the electrocardiogram (ECG). The board was then placed in a $38{ }^{\circ} \mathrm{C}$ water bath filled with degassed water which maintained the rat body temperature and provided coupling for ultrasound scanning and treatment. All rats were allowed to waken and had follow-up monitoring for 6 weeks. For follow-up echocardiography or other exams, the rats were anesthetized with $1-4 \%$ isoflurane in oxygen (Isotec 4, Surgivet Inc. Waukesha, WI USA), and scanned in the right lateral recumbent position on a warming pad.

\section{Ultrasound}

Ultrasound exposure for treatment was provided by a laboratory system with guidance by diagnostic ultrasound imaging, as described previously [14]. Briefly, the treatment system consisted of a function generator for generating a train of 10 cycle pulses at $4 \mathrm{kHz}$ PRF (model 3314A function generator, Hewlett Packard Co., Palo Alto CA, USA). An arbitrary waveform generator was used for amplitude modulation of the pulse train to give 8 pulse bursts (model 33220A, Agilent Technologies, Loveland CO, USA). A power amplifier (A-500, Electronic Navigation Industries, Rochester NY, USA) elevated the signal for driving the transducer. The rat ECG signal was amplified (Model ECGA amplifier, Hugo Sachs Elektronik, Harvard Apparatus, March, Germany) and displayed on an oscilloscope (Model TDS 520 B, Tektronix Inc., Beaverton, OR, USA), which was used to trigger the pulse bursts at end of systole. A $1.5 \mathrm{MHz}$ single element transducer (Panametrics A3464, Olympus, Waltham, MA, USA) with $1.9 \mathrm{~cm}$ diameter and $3.8 \mathrm{~cm}$ focal length was used for the treatment. The pulse exposure parameters were measured with a calibrated hydrophone (model HMA0200 membrane hydrophone, Onda Corp. Sunnyvale CA USA), and used to adjust the function generator voltage to generate a $4 \mathrm{MPa}$ peak rarefactional pressure amplitude (PRPA). The chest wall attenuation at $1.5 \mathrm{MHz}$ is about $1.1 \mathrm{~dB} / \mathrm{cm} / \mathrm{MHz}$ [20], which reduces the PRPA at the heart to about $3.6 \mathrm{MPa}$ after passing through the $5 \mathrm{~mm}$ thick chest wall. The equivalent in situ MI (the derated PRPA divided by the square-root of frequency) was therefore about 2.9;
Table 1 Parameters for the rats at initial anesthesia (average \pm standard deviation)

\begin{tabular}{llllll}
\hline Group & Treatment & $\mathrm{n}$ & $\mathrm{gm}$ & $\mathrm{BPM}$ & $\mathrm{SpO}_{2}$ \\
\hline A & $3 \times$ sham & 5 & $372 \pm 18$ & $265 \pm 23$ & $82 \pm 2$ \\
B & $3 \times 200 \mathrm{~s}$ & 6 & $391 \pm 12$ & $261 \pm 11$ & $90 \pm 4$ \\
C & $200 \mathrm{~s}$ & 6 & $405 \pm 20$ & $246 \pm 14$ & $85 \pm 5$ \\
D & $600 \mathrm{~s}$ & 6 & $395 \pm 29$ & $253 \pm 18$ & $86 \pm 4$ \\
\hline
\end{tabular}

The groups of rats were very similar, with some variation in weight due to the experimental plan having a staggered start (rats differing in age by up to 3 weeks)

for reference, the regulatory upper limit on the MI for diagnostic ultrasound is 1.9. The $-6 \mathrm{~dB}$ beam diameter was measured at the focus to be $3.5 \mathrm{~mm}$. The focus was targeted to the anterior surface of the heart with the aid of $10 \mathrm{MHz}$ diagnostic ultrasound imaging (GE Vivid 7 with $10 \mathrm{~S}$ probe, General Electric Corp., Cincinnati OH, USA), as previously described [14]. Treatments were either $200 \mathrm{~s}$ or $600 \mathrm{~s}$ in duration.

\section{Ultrasound contrast agent}

A continuous infusion of a microbubble suspension was given during treatment. The suspension was prepared to closely duplicate the ultrasound contrast agent Definity (Lantheus Medical Imaging, Inc., $\mathrm{N}$. Billerica, MA), as described previously [17, 21]. This suspension served as the source of in vivo cavitation nuclei for MCET, and contained $3.7 \pm 0.3\left(10^{9}\right)$ microbubbles per $\mathrm{mL}$ of $1.8 \pm 0.11 \mu \mathrm{m}$ diameter. This was diluted in sterile saline in a $5 \mathrm{ml}$ syringe, and infused via tail vein with a syringe pump at a rate providing $5 \mu \mathrm{l} / \mathrm{kg} / \mathrm{min}$ of agent.

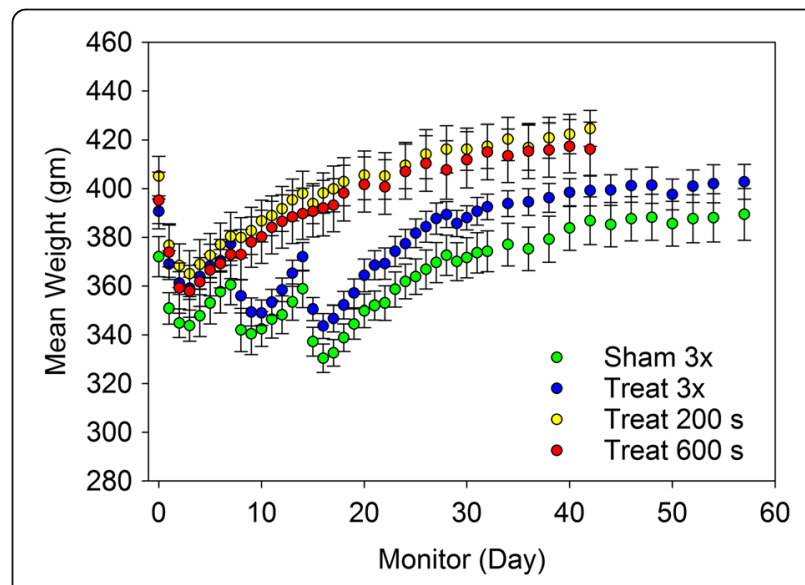

Fig. 1 Trends in the mean body weight for each of the groups. The steroid treatment induced a brief period of weight loss for each treatment session so that the fractionated treatment groups, both sham and exposed, had three brief periods of weight loss 
Table 2 Results for the percentage of pulse triggers resulting in premature complexes (PCs) in the ECG, and for the plasma troponin at $4 \mathrm{~h}$ (average \pm standard deviation)

\begin{tabular}{|c|c|c|c|c|c|c|c|c|}
\hline \multirow[b]{2}{*}{ Group } & \multicolumn{4}{|c|}{ Premature Complexes (\%) } & \multicolumn{4}{|c|}{ Plasma Troponin (ng/ml) } \\
\hline & $\operatorname{Exp} 1$ & $\operatorname{Exp} 2$ & Exp 3 & Mean & Exp 1 & $\operatorname{Exp} 2$ & Exp 3 & Total \\
\hline $\bar{A}$ & $0.2 \pm 0.5$ & $0 \pm 0$ & $0.4 \pm 0.9$ & $0.2 \pm 0.5$ & $0.3 \pm 0.02$ & $0.3 \pm 0.02$ & $0.48 \pm 0.03$ & $1.1 \pm 0.04$ \\
\hline B & $99 \pm 2$ & $97 \pm 2$ & $97 \pm 5$ & $97 \pm 2$ & $11.1 \pm 3.3$ & $5.6 \pm 2.0^{c}$ & $6.0 \pm 1.4^{c}$ & $22.7 \pm 5.4$ \\
\hline C & $96 \pm 6$ & & & $96 \pm 6$ & $10.5 \pm 3.2$ & & & $10.5 \pm 3.2$ \\
\hline D & $94 \pm 5$ & $82 \pm 7^{a}$ & $76 \pm 10^{\dagger}$ & $85 \pm 10^{b}$ & $29.9 \pm 6.4$ & & & $29.9 \pm 6.4$ \\
\hline
\end{tabular}

PC results for the $600 \mathrm{~s}$ treatment were assessed for each $200 \mathrm{~s}$ period, for comparison to the fractionated treatments. Results for the multiple treatments were summed to give the total effect. PCs were significantly less than in the 1st period for the successive periods of the $600 \mathrm{~s}$ treatment $\left({ }^{\mathrm{a}}\right)$, and the mean was significantly less than the mean for the fractionated treatments $\left(^{b}\right)$. Troponin release was significantly reduced for the 2 nd and $3 r d$ of the fractionated treatments $\left({ }^{\mathrm{C}}\right.$ )

\section{Adjuvant treatment}

Adjuvant treatment with methylprednisolone (methylprednisolone acetate injectable suspension, DepoMedrol, Pfizer Inc. New York, NY USA) and losartan (Losartan Potassium, TEVA Pharmaceuticals USA, INC. North Wales, PA) was given to the rats to improve the treatment outcome. Roberts et al. showed that initial inflammation and subsequent scarring from myocardial infarction in rats was reduced by high dose methylprednisolone treatment [22]. For MCET, methylprednisolone reduced the inflammatory response to microlesion injury and the swelling of the treated area [18]. Doses of $1 \mathrm{mg} /$ $\mathrm{kg}$ were given at treatment and at 4 and $24 \mathrm{~h}$ post treatment. The steroid treatment had a negative effect on the weight of the rats, and weights were measured at treatment and each day of follow-up. de Carvalho Frimm et al. reported that losartan, an angiotensin II receptor antagonist used for blood pressure control, reduced infarct size and collagen volume fraction (indicative of scarring) at 4 weeks post infarct [23]. For this study, losartan was given daily by gavage at $10 \mathrm{mg} / \mathrm{kg}$ in water, starting 1 day after ultrasound treatment and continuing for until the conclusion of the study.

\section{Measured endpoints}

Plasma troponin was measured in samples obtained $4 \mathrm{~h}$ after treatment, which provided a general measure of overall heart injury. Plasma troponin I (CTNI-2-HSP, Rat Cardiac Troponin-1 ELISA kit, Life Diagnostics Inc. West Chester, PA USA) is a sensitive measure of cardiac injury in rodents with essentially zero background in controls [24]. In previous work, the plasma troponin correlated with wall thinning observed in HCM model rats at $4 \mathrm{w}$ post treatment [18]. The cumulative release of troponin is a useful measure of total injury [25].

Left ventricular wall thickness and ejection fraction was measured by echocardiography at treatment (D0), the next day (D1) to assess the inflammatory increase in wall thickness, after 1 week (D7), and at euthanasia. In addition, the local reduction in kinetic motions of heart wall was measured by echocardiography using image analysis software (EchoInsight, Epsilon Imaging, Ann Arbor MI USA) as described previously [18]. The circumferential strain fraction (elasticity) and the displacement of the endocardium from diastole to systole were evaluated. For repeated measurements, the treated region could be identified accurately by the acoustic window and by hyperechogenicity in the ultrasound image (caused by the inflammatory response or fibrosis for later exams). These were compared to matched regions in sham treated hearts.

The healing and scar formation was also evaluated histologically, as described previously [18]. The hearts were fixed in an end-diastolic configuration [26], giving with dimensions approximating the dimensions observed by echocardiography. Histological slides were processed at the Histology Core of the University of Michigan Dental School with Masson's trichrome staining to show fibrosis. The wall thickness change was estimated relative to shams. In addition, the approximate percentage

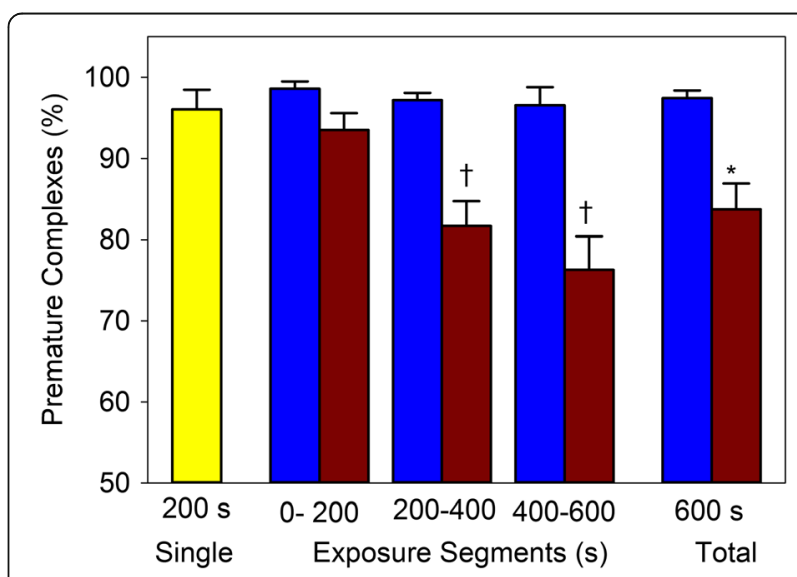

Fig. 2 Results for the percentage of pulse triggers resulting in premature complexes in the ECG (average \pm standard deviation) for $200 \mathrm{~s}$ (yellow), $600 \mathrm{~s}$ (red) and fractionated treatment (blue). PCs were significantly less than in the 1st period for the successive periods of the $600 \mathrm{~s}$ treatment $(t)$, and the total was significantly less than the total for the fractionated treatments $\left(^{*}\right)$ 
Table 3 Results for treatment impact given as the baseline compared to end results for the final exam before sacrifice (mean \pm standard deviation)

\begin{tabular}{|c|c|c|c|c|c|c|c|c|c|c|}
\hline \multirow[b]{2}{*}{ Group } & \multicolumn{2}{|c|}{ Echo wall (mm) } & \multicolumn{2}{|c|}{ Ejection Fraction } & \multicolumn{2}{|c|}{ Echo Strain (\%) } & \multicolumn{2}{|c|}{ Displace. (mm) } & \multirow{2}{*}{$\begin{array}{l}\text { Histo wall } \\
\mathrm{mm}\end{array}$} & \multirow{2}{*}{$\begin{array}{l}\text { Fibrosis } \\
\text { Area \% }\end{array}$} \\
\hline & Baseline & End & Baseline & End & Baseline & End & Baseline & End & & \\
\hline $\bar{A}$ & $1.7 \pm 0.1$ & $1.7 \pm 0.1$ & $80 \pm 3$ & $77 \pm 1$ & $51 \pm 7$ & $46 \pm 4$ & $0.88 \pm 0.13$ & $0.76 \pm 0.08$ & $2.3 \pm 0.4$ & 0 \\
\hline B & $1.7 \pm 0.1$ & $2.0 \pm 0.1^{* *}$ & $79 \pm 1$ & $75 \pm 2^{* *}$ & $45 \pm 8$ & $30 \pm 5^{* *}$ & $0.92 \pm 0.06$ & $0.76 \pm 0.08^{*}$ & $2.4 \pm 0.2$ & $40 \pm 10^{a}$ \\
\hline C & $1.8 \pm 0.1$ & $1.9 \pm 0.2^{*}$ & $80 \pm 4$ & $77 \pm 5$ & $46 \pm 5$ & $36 \pm 8^{*}$ & $0.87 \pm 0.08$ & $0.84 \pm 0.11$ & $2.4 \pm 0.4$ & $29 \pm 16^{a}$ \\
\hline D & $1.9 \pm 0.2$ & $1.5 \pm 0.2^{* *}$ & $79 \pm 3$ & $65 \pm 6^{* *}$ & $45 \pm 7$ & $25 \pm 7^{* *}$ & $0.88 \pm 0.08$ & $0.54 \pm 0.11^{* *}$ & $1.8 \pm 0.3^{\mathrm{a}}$ & $49 \pm 8^{a}$ \\
\hline
\end{tabular}

Significant differences versus baseline denoted by ${ }^{*}$ for $p<0.05$ or ${ }^{* *}$ for $p<0.01$. Significant difference versus sham (A) denoted by ${ }^{a}$. In addition, the fibrosis was higher in $D$ than in $C(p<0.05)$, but there was no significant differences for the other comparisons (B versus $C$, and $B$ versus $D)$

of the treated areas with fibrosis was assessed by image analysis of blue Masson's staining.

\section{Experimental plan}

Twenty-four Dahl SS rats, 20-22 weeks of age, were used for this study in 4 groups, listed in Table 1 . The groups were: A. sham multiple treatment (5 rats), B. three $200 \mathrm{~s}$ treatments (6 rats), C. single $200 \mathrm{~s}$ treatment (6 rats) and D. single $600 \mathrm{~s}$ treatment (6 rats). One rat died in animal housing before use, which was subtracted from the sham group. One rat from the multi-treatment group died $35 \mathrm{~d}$ after beginning treatment, with that final datum lost from the data set. Rats were randomly chosen for each group. Four rats were used each of 12 treatment days, which were selected from the groups in a defined pattern to balance the order of handling in each group. This was complicated by the multiple treatments. Each treatment day, 3 rats received one of their multiple treatments (alternating $1 \mathrm{~A}$ and $2 \mathrm{~B}$ with $1 \mathrm{~B}$ and $2 \mathrm{~A}$ ), and one rat received its single treatment (alternating group $\mathrm{C}$ or $\mathrm{D}$ ).

Results are reported as the means plus or minus one standard deviation, or plotted with standard error bars. Statistical analysis was performed (SigmaPlot for Windows V. 11.0, Systat Software Inc., San Jose CA, USA). Student's $t$-tests were used to compare means of the measured parameters, with statistical significance assumed at $p<0.05$.

\section{Results}

The physiological parameters for the groups are listed in Table 1. All had approximately the same weight, heart rate under anesthesia and $\mathrm{SpO}_{2}$ under anesthesia. The rats all received the steroid treatment at each exposure session. Therefore the fractionated exposure with three $200 \mathrm{~s}$ exposures had 3 dose regimens of the steroid. The steroid treatment produced a brief period of weight reduction in the Dahl SS rats, considered to be an adverse reaction. The weight trends for each group are plotted in Fig. 1. The fractionated treatment groups (sham and exposed) had quite similar trends of repeated brief weight loss, which indicates the weight changes were due to the steroid, and not the MCET treatment.

The results for PCs and troponin release at each treatment are listed in Table 2. The PCs for the $600 \mathrm{~s}$ group are broken down into $200 \mathrm{~s}$ segments for comparison to the fractionated treatment group, as shown in Fig. 2. For the fractionated treatment groups, the results for the three treatments are also totaled for comparison to the $600 \mathrm{~s}$ group. There was a significant trend for the PCs to decline during the $600 \mathrm{~s}$ exposures such that the 2nd and 3rd $200 \mathrm{~s}$ periods had significantly fewer PCs than the first period, and the total was significantly less than for the fractionated treatment. Interestingly, although PCs remained the same $(\geq 97 \%)$, troponin release for the 2 nd and $3 r d$ fractions of the fractionated treatment, which were about the same, were significantly less than the first fraction $(p<0.01)$. The troponin total for the fractionated treatment was somewhat less than for the single $600 \mathrm{~s}$ treatment, but the difference was not significant $(p=0.06)$.

The results of the follow-up monitoring are listed in Table 3 for the baseline and end (i. e. the final exam

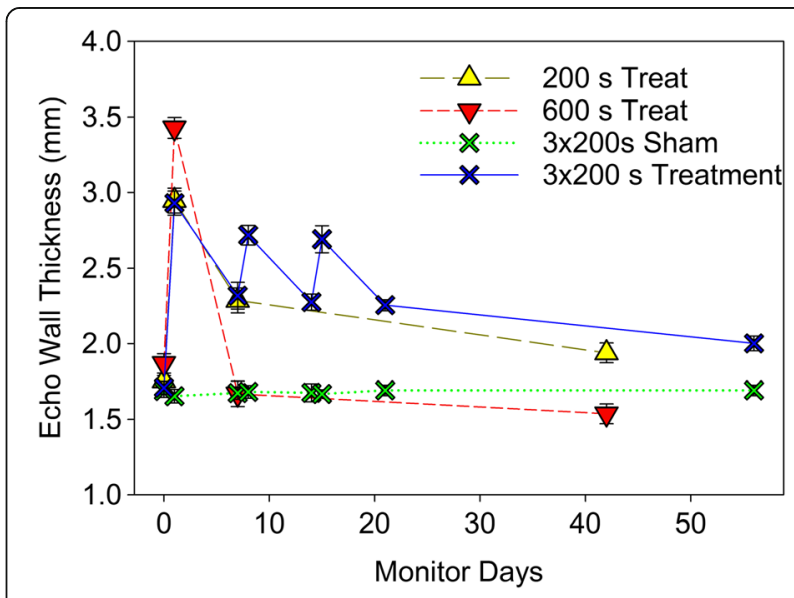

Fig. 3 The trends in echocardiographic wall thickness for the groups. Treatment induced immediate swelling which was notable the next day, then receded after 1 week and at the end of monitoring. Only the 600 s treatment gave a significant reduction in wall thickness $(p<0.01)$ 
before sacrifice) echocardiographic wall thickness, ejection fraction, wall strain and endocardial displacement determined from the echocardiographic images, the wall thickness measured in histological samples, and the percentage of fibrotic area seen in Masson's trichrome slides within the target region. The results were significantly different from baseline for all treated groups. The treatment caused wall swelling after 1 day, which decreased for subsequent measurements at 1 week and at the end of the monitoring. These trends are shown in Fig. 3. For the fractionated treatments, the 3 episodes of swelling and recovery are clearly apparent. Of note, the $600 \mathrm{~s}$ treatment group had the wall thickness recede to pre-treatment dimensions, equal to the sham values, after 1 week. The final wall thickness was significantly increased for the $200 \mathrm{~s}$ and fractionated treatment, but significantly reduced for the $600 \mathrm{~s}$ treatment.

The heart samples showed visual evidence of fibrosis, as shown in Fig. 4. The fibrosis had a characteristic white circular appearance which corresponded to the beam target area. In addition, for the $600 \mathrm{~s}$ groups, an extra region of fibrosis was sometimes clearly evident toward the upper right of the target area; the clearest example is shown in the figure. In histology, the fibrosis, stained blue in Masson's trichrome histology (Fig. 4), was evident as scattered microlesion regions in the $200 \mathrm{~s}$ and fractionated exposures. In the $600 \mathrm{~s}$ treatment shown, the section reveals a thin region of tissue erosion on the anterior surface (possibly, giving the very bright white appearance on the heart surface).

\section{Discussion}

MCET produces scattered microlesions encompassing a few lethally injured cardiomyocytes, which accumulate with each triggered pulse burst. The total "macrolesion" can be sufficient for therapy of hypertrophic cardiomyopathy both in terms of heart tissue volume reduction and in akinesis of wall motion and strain. Previous work with Dahl SS rats had demonstrated substantial tissue impact, with minimal impact on heart function after healing for single long (600 s) treatments [17, 18]. Adjuvant treatment with methylprednisolone and losartan reduce the initial swelling and aid in reduction of fibrosis. In this study, the strategy of fractionated treatment which could allow graded treatment and retreatment adjustments was assessed in normal Dahl SS rats. This strategy is an important distinction from surgical myectomy and alcohol trans-catheter ablation, the currently accepted treatments, because those methods are essentially single conclusive treatments.

This study demonstrated that repeated treatments were well tolerated and produced incremental increases in effect as measured by troponin release. However, the 2nd and 3rd treatments a week apart were less effective than the first. The treatment did not produce a reduction in wall thickness, which remained greater than the baseline (Fig. 3). However, the akinesis produced by the fractionated treatment was significant. Akinesis induced by alcohol ablation appears to be responsible for the immediate relief of symptoms [10], and should be therapeutic also for the MCET method.

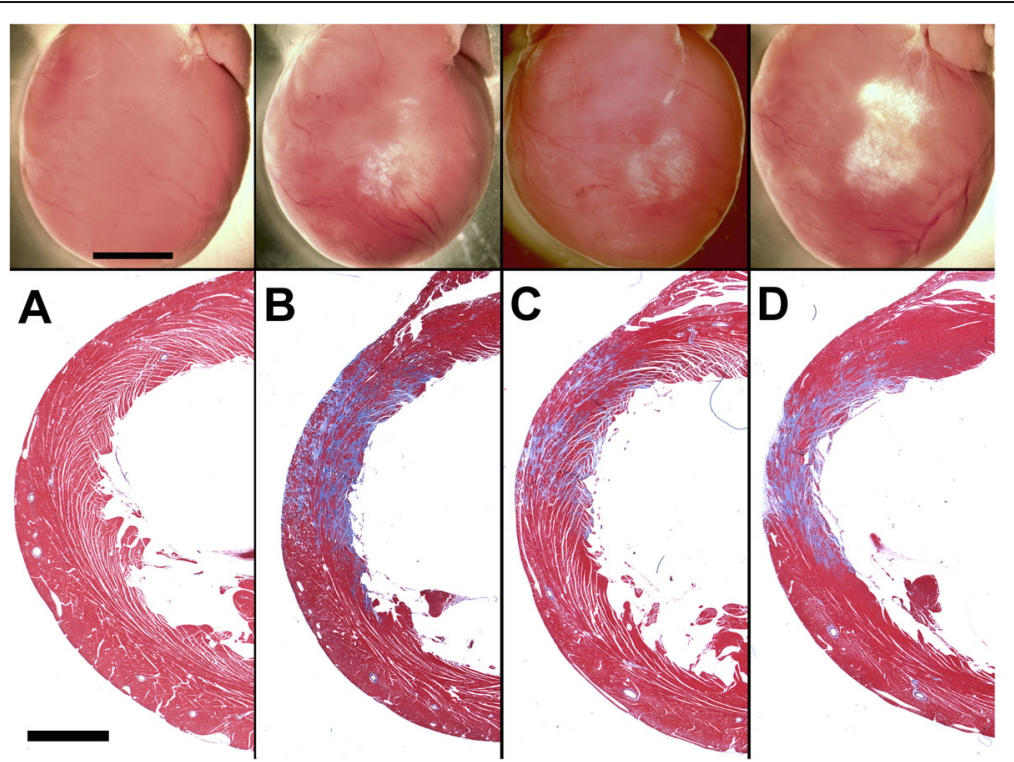

Fig. 4 Selected examples of the hearts for each group. Photographs are shown of hearts for groups A-D (scale bar 5 mm), and corresponding histological sections (scale bar $2 \mathrm{~mm}$ ) stained with Masson's trichrome to reveal fibrosis (blue). For the $600 \mathrm{~s}$ treatment (D), the wall is noticeably thinned in the target area, including some erosion of the heart surface 
The single $600 \mathrm{~s}$ treatment, used for previous chronic studies $[17,18]$, was remarkably effective in this study with a $21 \%$ reduction in wall thickness (Table 3 ). This effectiveness may have included a secondary mechanism of injury. The heart shown in Fig. 4 from group D has an exaggerated fibrotic region which appears to be outside the targeted region. For the fractionated treatment, the real time indicator of PCs was the same for the 3 fractions, but declined for each $200 \mathrm{~s}$ segment of the $600 \mathrm{~s}$ treatment, while apparently yielding greater impact. One possible explanation for the extra fibrosis region is heart motion due to breathing, which periodically could shift the target point of the ultrasound beam. However, the histologically observed result (Fig. 4) seemed to be greater than within the target region, and included a surface layer of myocardial ablation not unlike that seem in ischemia induced infarcts. Thus, another explanation for the high efficacy of the $600 \mathrm{~s}$ treatment may be local ischemia resulting from the tissue swelling or microvascular occlusion. This possibility should be investigated and minimized to allow better treatment control for the desired outcome of scattered microlesions within a functional treated volume.

\section{Conclusions}

In conclusion, the strategy of MCET treatment fractionation was shown to be feasible, and represents a potential advantage relative to currently accepted therapy. A possible adverse effect of partial ablation was seen for a $600 \mathrm{~s}$ treatment, not previously noted for MCET. However, this was not noted for the fractionated treatment. An unexpected contrast was found for the resulting left ventricular wall thickness, which was increased for fractionated and decreased for continuous treatment. Previously, wall thickness reduction was not seen for normal Dahl SS rats [17] without adjuvant treatment but was seen for the SS-16 ${ }^{\mathrm{BN}}$ rat model of $\mathrm{HCM}$ with the adjuvant treatments [18]. Further study of the safety and efficacy of MCET is needed, especially for larger animal models such as cats [27], which have a more clinically relevant presentation of $\mathrm{HCM}$.

\section{Acknowledgments}

Not applicable.

\section{Funding}

Research reported in this publication was supported by the National Heart Lung and Blood Institute of the National Institutes of Health under award number R01HL114595. The content is solely the responsibility of the authors and does not necessarily represent the official views of the National Institutes of Health.

\section{Availability of data and materials}

The datasets used and/or analyzed during the current study are available from the corresponding author on reasonable request.

\section{Authors' contributions}

The experimental design, data acquisition and interpretation were performed by all authors. An initial draft manuscript was prepared by DLM and revised by $\mathrm{XL}, \mathrm{GEO}$ and GDK. All authors read and approved the final manuscript.

\section{Ethics approval}

All in vivo animal procedures were conducted with the approval and guidance of the University Committee on Use and Care of Animals of the University of Michigan.

\section{Consent for publication}

Not applicable.

\section{Competing interests}

The authors declare that they have no competing interests.

\section{Publisher's Note}

Springer Nature remains neutral with regard to jurisdictional claims in published maps and institutional affiliations.

\section{Author details}

${ }^{1}$ Department of Radiology, University of Michigan School of Medicine, Ann Arbor, Ml 48109, USA. ${ }^{2}$ Department of Pediatrics (Cardiology), University of Michigan School of Medicine, Ann Arbor, MI 48109, USA. ${ }^{3}$ University of Michigan School of Medicine, 3240A Medical Sciences Building I, 1301 Catherine Street, Ann Arbor, Ml 48109-5667, USA.

Received: 1 August 2017 Accepted: 19 October 2017

Published online: 09 November 2017

\section{References}

1. Semsarian C, Ingles J, Maron MS, Maron BJ. New perspectives on the prevalence of hypertrophic cardiomyopathy. J Am Coll Cardiol. 2015:65:1249-54.

2. Maron BJ, Salberg L. Wiley online, a guide to hypertrophic cardiomyopathy for patients, families, and interested physicians. Chichester, West Sussex: John Wiley \& Sons Ltd.; 2014

3. Ommen SR, Nishimura RA. Hypertrophic cardiomyopathy. Curr Probl Cardiol. 2004;29:239-91.

4. Marian AJ. Contemporary treatment of hypertrophic cardiomyopathy. Tex Heart Inst J. 2009;36:194-204.

5. Jan MF, Todaro MC, Oreto L, Tajik AJ. Apical hypertrophic cardiomyopathy: present status. Int J Cardiol. 2016:222:745-59.

6. Maron BJ, McKenna WJ, Danielson GK, Kappenberger LJ, Kuhn HJ, Seidman CE, Shah PM, Spencer WH 3rd, Spirito P, Ten Cate FJ, Wigle ED. Task force on clinical expert consensus documents. American College of Cardiology; Committee for Practice Guidelines. European Society of Cardiology. American College of Cardiology/European Society of Cardiology clinical expert consensus document on hypertrophic cardiomyopathy. A report of the American College of Cardiology Foundation task force on clinical expert consensus documents and the European Society of Cardiology Committee for practice guidelines. J Am Coll Cardiol. 2003;42:1687-713.

7. Gersh BJ, Maron BJ, Bonow RO, Dearani JA, Fifer MA, Link MS, Naidu SS, Nishimura RA, Ommen SR, Rakowski H, Seidman CE, Towbin JA, Udelson JE, Yancy CW. 2011 ACCF/AHA guideline for the diagnosis and treatment of hypertrophic Cardiomyopathy: a report of the American College of Cardiology Foundation/American Heart Association task force on practice guidelines. Developed in collaboration with the American Association for Thoracic Surgery, American Society of Echocardiography, American Society of Nuclear Cardiology, Heart Failure Society of America, Heart Rhythm Society, Society for Cardiovascular Angiography and Interventions, and Society of Thoracic Surgeons. J Am Coll Cardiol. 2011;58(25):e212-60.

8. Brown ML, Schaff HV, Dearani JA, Li Z, Nishimura RA, Ommen SR. Relationship between left ventricular mass, wall thickness, and survival after subaortic septal myectomy for hypertrophic obstructive cardiomyopathy. J Thorac Cardiovasc Surg. 2011;141:439-43.

9. Leonardi RA, Kransdorf EP, Simel DL, Wang A. Meta-analyses of septal reduction therapies for obstructive hypertrophic cardiomyopathy: comparative rates of overall mortality and sudden cardiac death after treatment. Circ Cardiovasc Interv. 2010;3:97-104. 
10. Dąbrowski M, Chojnowska L, Małek L, Spiewak M, Kuśmierczyk B, Koziarek J, Klisiewicz A, Miśko J, Witkowski A. An assessment of regression of left ventricular hypertrophy following alcohol ablation of the interventricular septum in patients with hypertrophic cardiomyopathy with left ventricular outflow tract obstruction. Kardiol Pol. 2012;70:782-8.

11. Baggish AL, Smith RN, Palacios I, Vlahakes GJ, Yoerger DM, Picard MH, Lowry PA, Jang IK, Fifer MA. Pathological effects of alcohol septal ablation for hypertrophic obstructive cardiomyopathy. Heart. 2006;92:1773-8.

12. Nishimura RA, Ommen SR. Septal reduction therapy for obstructive hypertrophic cardiomyopathy and sudden death: what statistics cannot tell you. Circ Cardiovasc Interv. 2010;32:91-3.

13. Miller DL, Li P, Dou C, Gordon D, Edwards CA, Armstrong WF. Influence of contrast agent dose and ultrasound exposure on cardiomyocyte injury induced by myocardial contrast echocardiography in rats. Radiology. 2005;237:137-43.

14. Miller DL, Dou C, Owens GE, Kripfgans OD. Optimization of ultrasound parameters of myocardial cavitation microlesions for therapeutic applications. Ultrasound Med Biol. 2014;40:1228-36.

15. Zhu Yl, Miller DL, Dou C, Lu X, Kripfgans OD. Quantitative assessment of damage during MCET: a parametric study in a rodent model. J Ther Ultrasound. 2015;3:18.

16. Miller DL, Dou C, Owens GE, Kripfgans OD. Timing of high-intensity pulses for myocardial cavitation-enabled therapy. J Ther Ultrasound. 2014;2:20.

17. Lu X, Miller DL, Dou C, Zhu YI, Fabiilli ML, Owens GE, Kripfgans OD. Maturation of lesions induced by myocardial Cavitation-enabled therapy. Ultrasound Med Biol. 2016:42:1541-50.

18. Miller DL, Lu X, Dou C, Zhu Yl, Fuller R, Fields K, Fabiilli ML, Owens GE, Gordon D and Kripfgans OD. Ultrasonic Cavitation-Enabled Treatment for Therapy of Hypertrophic Cardiomyopathy: Proof of Principle. Ultrasound Med. Biol. (in press).

19. Kriegel AJ, Greene AS. Substitution of Brown Norway chromosome 16 preserves cardiac function with aging in a salt-sensitive Dahl consomic rat. Physiol Genomics. 2008;36:35-42.

20. Miller DL, Dou C, Raghavendran K. Dependence of thresholds for pulmonary capillary hemorrhage on diagnostic ultrasound frequency. Ultrasound Med Biol. 2015;41:1640-50.

21. Miller DL, Lu X, Fabiilli M, Dou C. Do anesthetic techniques influence the threshold for glomerular capillary hemorrhage induced in rats by contrastenhanced diagnostic ultrasound? J Ultrasound Med. 2016:35:373-80.

22. Roberts CS, Maclean D, Maroko P, Kloner RA. Relation of early mononuclear and polymorphonuclear cell infiltration to late scar thickness after experimentally induced myocardial infarction in the rat. Basic Res Cardiol. 1985;80:202-9.

23. De Carvalho Frimm C, Sun Y, Weber KT, Angiotensin II. Receptor blockade and myocardial fibrosis of the infarcted rat heart. J Lab Clin Med. 1997;129:439-46.

24. O'Brien PJ, Smith DE, Knechtel TJ, Marchak MA, Pruimboom-Brees I, Brees DJ, Spratt DP, Archer FJ, Butler P, Potter AN, Provost JP, Richard J, Snyder PA, Reagan WJ. Cardiac troponin I is a sensitive, specific biomarker of cardiac injury in laboratory animals. Lab Anim. 2006:40:153-71.

25. Kragten JA, Hermens WT, Van Dieijen-Visser MP. Cumulative troponin $T$ release after acute myocardial infarction. Influence of reperfusion. Eur I Clin Chem Clin Biochem. 1997;35:459-67.

26. Whittaker $P$, Patterson MJ. Ventricular remodeling after acute myocardial infarction: effect of low-intensity laser irradiation. Lasers Surg Med. 2000;27:29-38.

27. Kittleson MD, Meurs KM, Munro JM, Kittleson JA, Liu SK, Pion PD, Towbin JA. Familial hypertrophic cardiomyopathy in Maine coon cats: an animal model of human disease. Circulation. 1999;99:3172-80.

\section{Submit your next manuscript to BioMed Central and we will help you at every step:}

- We accept pre-submission inquiries

- Our selector tool helps you to find the most relevant journal

- We provide round the clock customer support

- Convenient online submission

- Thorough peer review

- Inclusion in PubMed and all major indexing services

- Maximum visibility for your research

Submit your manuscript at www.biomedcentral.com/submit
Biomed Central 